\title{
Avaliação antropométrica e bioquímica em pacientes com esquizofrenia usuários de clozapina
}

\author{
Anthropometric and biochemical assessment \\ of schizophrenic patients taking clozapine
}

Karine ZORTÉA ${ }^{1}$

Patrícia Martins BOCK²

Dolores Benites MORENO ${ }^{1}$

Paulo Silva Belmonte de ABREU1

RE S U M O

\section{Objetivo}

Avaliar a proporção de sobrepeso/obesidade e alterações em parâmetros bioquímicos sangüíneos em pacientes com esquizofrenia do sexo masculino, usuários de medicação antipsicótica (clozapina).

\section{Métodos}

Estudo transversal com 40 pacientes do sexo masculino, diagnosticados com esquizofrenia, em uso de clozapina, atendidos no Hospital de Clínicas de Porto Alegre. Foram verificadas as relações entre o índice de massa corporal, a circunferência abdominal, o percentual de gordura corporal, o perfil lipídico e o exame de glicose de jejum e a dosagem da medicação, o acompanhamento dietoterápico e a monoterapia de clozapina.

\section{Resultados}

Os pacientes apresentaram freqüências de 71,8\% de sobrepeso/obesidade, 76,9\% de circunferência abdominal aumentada e $94,1 \%$ de percentual de gordura elevada. Foram encontrados $56,8 \%$ de pacientes com lipoproteína de baixa densidade-colesterol acima do normal e 64,1\% com triglicerídeos elevados. Quanto maior a dosagem de clozapina utilizada maiores foram os níveis de lipoproteína de baixa densidade-colesterol $(p=0,01)$.

\section{Conclusão}

Pacientes tratados com clozapina apresentam aumento de peso e significante anormalidade lipídica, necessitando de monitoramento freqüente dos níveis plasmáticos e intervenção nutricional precoce, visando à otimização do tratamento.

Termos de indexação: Clozapina. Esquizofrenia. Obesidade.

\footnotetext{
${ }^{1}$ Universidade Federal do Rio Grande do Sul, Departamento de Psiquiatria do Hospital de Clínicas de Porto Alegre. R. Ramiro Barcelos, 2350, Santa Cecília, 90035-903, Porto Alegre, RS, Brasil. Correspondência para/Correspondence to: K. ZORTÉA. E-mail: <karine.personaldiet@gmail.com>.

2 Centro Universitário Metodista. Porto Alegre, RS, Brasil.
} 
698 | K. ZORTÉA et al.

\section{A B S T R A C T}

\section{Objective}

The objective was to access the overweight/obesity rates and variations of biochemical blood parameters in male schizophrenic outpatients taking antipsychotic medication (clozapine), treated at the Schizophrenia and Dementia First Aid Station of the Clinics Hospital in Porto Alegre.

\section{Methods}

This is a cross-sectional study with 40 male outpatients diagnosed with schizophrenia and taking clozapine, from the Clinics Hospital in Porto Alegre. Associations between body mass index, waist circumference, body fat percentage, lipid levels and fasting glucose and medication dosage, diet therapy and clozapine monotherapy were verified.

\section{Results}

There was a rate of $71.8 \%$ of overweight/obesity, $76.9 \%$ of increased waist circumference and $94.1 \%$ of high body fat percentage. More than half of the patients (56.8\%) had low density lipoprotein cholesterol levels above normal and $64.1 \%$ had high triglycerides. LDL-cholesterol levels increased with increasing clozapine dose $(p=0.01)$.

\section{Conclusion}

Patients on clozapine presented excess weight and significant lipid abnormality, needing frequent monitoring of the biochemical blood parameters and early nutritional intervention to optimize treatment.

Indexing terms: Clozapine. Schizophrenia. Obesity.

\section{N T R O D U Ç Ã O}

A esquizofrenia caracteriza-se como uma perturbação presente, no mínimo, há seis meses incluindo ao menos um mês de sintomas da fase ativa, como delírios, alucinações, sintomas negativos, discurso e comportamento desorganizados. O diagnóstico envolve o reconhecimento de um conjunto de sinais e sintomas associados ao prejuízo do funcionamento ocupacional ou social ${ }^{1}$, como o eco, imposição, roubo ou divulgação do pensamento, percepção delirante, idéias delirantes de controle, vozes alucinatórias que conversam com o paciente, entre outros ${ }^{2}$.

Estudos de prevalência sugerem que em torno de $1 \%$ da população brasileira apresenta esquizofrenia ${ }^{3,4}$, sendo a incidência de 1 a 7 casos novos por ano a cada 10 mil habitantes, dependendo do critério diagnóstico adotado ${ }^{3}$. Há igual prevalência entre homens e mulheres, porém os sexos apresentam divergências no início e no curso da doença ${ }^{5}$. Mulheres têm um prognóstico melhor do que os homens em relação ao número de reinternações psiquiátricas, à evolução clínica e ao desenvolvimento social. O suicídio também é mais freqüente entre os homens e está associado à depressão e à falta de motivação durante a vida6 .

O tratamento da esquizofrenia tem como ênfase o uso de medicamentos antipsicóticos, que incluem três classes principais: antagonistas dos receptores dopamínicos, risperidona e clozapina ${ }^{5}$.

A clozapina é um antipsicótico atípico de referência, por ter características distintas das medicações disponíveis antes de seu lançamento, que mostrou eficácia em 30 a $60 \%$ das psicoses que não respondem às drogas clássicas, e baixa incidência de efeitos adversos ${ }^{7}$, sendo, então, a principal indicação para a esquizofrenia refratária?. Os atípicos oferecem várias vantagens importantes sobre os típicos, incluindo suas características de menor risco de sintomas extrapiramidais, como acatisia, distonia, parkinsonismo e discinesia tardia ${ }^{4,8-10}$, melhora da cognição, suicídio reduzido e menor depressão ${ }^{11}$, quando comparados com os antipsicóticos típicos em doses clinicamente relevantes.

Apesar de os antipsicóticos atípicos serem considerados fármacos importantes no tratamento de psicoses, podem provocar alterações no peso 
e na homeostase dos níveis de glicose e lipídios, necessitando de intervenção precoce para reduzir estes efeitos, enquanto mantêm os benefícios clínicos ${ }^{11,12}$.

A obesidade é um fator de risco para doenças como diabetes, hipertensão arterial, dislipidemias e circunferência abdominal aumentada. O agrupamento de três ou mais destes fatores pode resultar na condição conhecida como Síndrome Metabólica ${ }^{13,14}$. Sugere-se que a clozapina pode contribuir para o surgimento de hiperinsulinemia e Síndrome Metabólica ${ }^{12}$.

Com bases nos efeitos combinados de estilo de vida, história familiar, efeitos relatados pela doença psiquiátrica, e eventos adversos do tratamento, muitos pacientes, atualmente, apresentam fatores de risco cardiovasculares múltiplos, incluindo tabagismo, obesidade, hiperglicemia e hipertrigliceridemia ${ }^{15}$. A meta da farmacoterapia é maximizar os benefícios e minimizar efeitos adversos, por isso, uma compreensão do monitoramento apropriado para efeitos adversos metabólicos é importante para aqueles que prescrevem antipsicóticos atípicos. A tarefa de prevenir os efeitos adversos pode ser mais fácil e mais efetiva do que tratar esses eventos cada vez em que ocorrem ${ }^{11}$.

Este estudo teve como objetivos avaliar a ocorrência de sobrepeso/obesidade e alterações em parâmetros bioquímicos sangüíneos em pacientes portadores de esquizofrenia, e identificar sua relação com a medicação utilizada.

\section{MÉ T O D O S}

A população estudada compreendeu homens com idade superior a 18 anos, com diagnóstico de esquizofrenia, de acordo com DSM-IV ${ }^{1}$ e o CID-102. Todos eram usuários de medicação antipsicótica (clozapina) no mínimo há três meses, e freqüentadores do Ambulatório de Esquizofrenia e Demências do Hospital de Clínicas de Porto Alegre (HCPA).

Trata-se de um estudo transversal no qual foi utilizada uma amostra de 40 indivíduos, de acordo com cálculo estatístico. Foram excluídos do estudo mulheres, por apresentarem respostas diferentes em relação à medicação, pacientes em uso de medicação para normalizar o colesterol e a glicemia e pacientes com diagnóstico de diabetes.

Os dados foram coletados por meio de entrevistas realizadas com os pacientes que consentiram em participar da pesquisa, quando responderam oralmente a um questionário e tiveram seus dados antropométricos (peso, altura, circunferência abdominal, percentual de gordura por bioimpedância) medidos e exames sangüíneos (colesterol total, colesterol HDL - Lipoproteína de Alta Densidade (HDL-C), colesterol LDL - Lipoproteína de Baixa Densidade (LDL-C), triglicerídeos, glicose de jejum e insulina) solicitados.

As medidas antropométricas foram realizadas por nutricionistas da equipe. Para verificar o peso corporal, os indivíduos deveriam estar descalços, vestindo roupas leves. Foi utilizada uma balança digital antropométrica da marca Filizola, devidamente aferida, e a altura foi verificada com o auxílio de um estadiômetro. Com a utilização destes dados foi possível realizar a classificação do estado nutricional dos pacientes por meio de:

- Índice de Massa Corporal (IMC): baixo peso $\left(<18,49 \mathrm{~kg} / \mathrm{m}^{2}\right)$, eutrofia $\left(18,5-24,99 \mathrm{~kg} / \mathrm{m}^{2}\right)$, sobrepeso $\left(25-29,99 \mathrm{~kg} / \mathrm{m}^{2}\right)$ e obesidade $\left(\geq 30 \mathrm{~kg} / \mathrm{m}^{2}\right)^{16}$. O cálculo é realizado a partir da razão peso $(\mathrm{kg}) /$ altura² $(\mathrm{m})$.

- Circunferência abdominal, medida na metade da distância entre a crista ilíaca e o rebordo costal inferior ${ }^{13}$, com auxílio de fita métrica inelástica, tendo como unidade de referência até $94 \mathrm{~cm}$ - classificação normal e acima de $94 \mathrm{~cm}$ - circunferência abdominal elevada, para o sexo masculino ${ }^{17}$.

- Percentual de gordura corporal, por bioimpedância elétrica, com aparelho marca Omron BF 300 , classificado como: $\leq 5 \%$ risco nutricional; 6 14\% abaixo da média; $15 \%$ média; $16-24 \%$ acima da média; $\geq 25 \%$ risco para doenças associadas à obesidade, para o sexo masculino ${ }^{18}$. 
$700 \mid$ K. ZORTÉA et al.

Os pacientes, após jejum de 12h, tiveram $5 \mathrm{~mL}$ de sangue coletado no laboratório do HCPA, para posterior análise, no mesmo laboratório. A glicose, o colesterol total, os triglicerídeos e o HDL-c foram mensurados pelo método de enzimacolorometria. O LDL-c foi calculado pela equação de Friedwald (CT - HDL-c - TG/5). Os níveis séricos de insulina foram mensurados por ensaio imunométrico eletroquimioluminescente (Roche, E-170).

Os resultados foram avaliados baseados na classificação do Adult Treatment Panel III (ATP III) ${ }^{19}$ e da IV Diretriz Brasileira sobre Dislipidemias e Prevenção da Aterosclerose ${ }^{17}$, para colesterol (colesterol total abaixo de 200mg/dL; LDL-c abaixo de $100 \mathrm{mg} / \mathrm{dL} ; \mathrm{HDL}-\mathrm{c}$ acima de $40 \mathrm{mg} / \mathrm{dL}$; triglicerídeos abaixo de $150 \mathrm{mg} / \mathrm{dL}$ ) e na classificação da American Diabetes Association (ADA) ${ }^{20}$ para glicose (normal: glicemia de jejum entre $70 \mathrm{mg} / \mathrm{dL}$ e $99 \mathrm{mg} / \mathrm{dL}$; intolerância à glicose - entre $100 \mathrm{e}$ $125 \mathrm{mg} / \mathrm{dL}$; diabetes - igual ou superior a $126 \mathrm{mg} / \mathrm{dL}$ em duas amostras diferentes.

O estudo foi submetido à aprovação do Comitê de Ética e Pesquisa em Saúde do HCPA em 2005, como parte do projeto "Associação da adiponectina e obesidade em pacientes esquizofrênicos expostos à neurolépticos", sob o protocolo $n^{\circ}$ 04-503. Todos os pacientes assinaram o Termo de Consentimento Livre e Esclarecido, aceitando participar do estudo.

Os dados coletados estão apresentados de forma descritiva, com Média (M) e Desvio-Padrão (DP) ou freqüências. Para relacionar a dose da medicação, o IMC, a circunferência abdominal, o percentual de gordura e os exames sangüíneos foram utilizados o Teste Qui-quadrado, o Teste exato de Fisher e o Teste de Spearman. Todas as análises estatísticas foram realizadas com o auxílio do programa Statistical Package for the Social Sciences (SPSS), Versão 13.0. Um valor de $p<0,05$ foi considerado significante.

\section{RESULTADOS}

Os dados antropométricos (Tabela 1) demonstram que os pacientes apresentaram freqüência de $71,8 \%$ de excesso de peso (sobre- peso/obesidade), 76,9\% de circunferência abdominal acima do desejado e $94,1 \%$ de percentual de gordura corporal elevado. Em relação aos parâmetros bioquímicos 56,8\% apresentaram níveis de LDL-c acima da média e 64,1\% apresentaram triglicerídeos elevados. A média de IMC encontrada nos pacientes foi $M=27,81$ $\mathrm{DP}=3,9 \mathrm{~kg} / \mathrm{m}^{2}$.

Tabela 1. Classificação de pacientes esquizofrênicos em relação aos dados antropométricos e bioquímicos. Porto Alegre (RS), 2007.

\begin{tabular}{|c|c|}
\hline Variável & Classificação \\
\hline \multicolumn{2}{|l|}{ Dados antropométricos } \\
\hline IMC $\left(\mathrm{kg} / \mathrm{m}^{2}\right)$ & $M=27,8 \quad D P=3,9$ \\
\hline \multicolumn{2}{|l|}{ Estado nutricional (\%) } \\
\hline Baixo peso & 2,6 \\
\hline Eutrofia & 25,6 \\
\hline Sobrepeso & 46,2 \\
\hline Obesidade & 25,6 \\
\hline Circunferência abdominal (cm) & $M=102,9 \quad D P=13,7$ \\
\hline Acima de $94 \mathrm{~cm}$ & 23,1 \\
\hline Abaixo de $94 \mathrm{~cm}$ & 76,9 \\
\hline \% de Gordura corporal & $M=25,2 \quad D P=5,6$ \\
\hline Abaixo do normal ( $\leq 5 \%)$ & 5,9 \\
\hline Média (15\%) & 0 \\
\hline Acima da média (16-24\%) & 38,2 \\
\hline $\begin{array}{l}\text { Risco para doenças associadas à obesidade } \\
(\geq 25 \%)\end{array}$ & 55,9 \\
\hline \multicolumn{2}{|l|}{ Perfil bioquímico (mg/dL) } \\
\hline Colesterol total (\%) & $M=197,6 \quad D P=45,5$ \\
\hline$<200$ & 60,5 \\
\hline$\geq 200$ & 39,5 \\
\hline$L D L-C$ & $M=114,0 \quad D P=40,9$ \\
\hline$<100$ & 43,2 \\
\hline$\geq 100$ & 56,8 \\
\hline$H D L-c(\%)$ & $M=44,9 \quad D P=9,8$ \\
\hline$<40$ & 24,3 \\
\hline$\geq 40$ & 75,7 \\
\hline \multicolumn{2}{|l|}{ Triglicerídeos (\%) } \\
\hline$<150$ & 35,9 \\
\hline$\geq 150$ & 64,1 \\
\hline Glicemia de jejum (\%) & $M=96,9 \quad D P=9,8$ \\
\hline$<100$ & 66,7 \\
\hline$\geq 100$ & 33,3 \\
\hline
\end{tabular}

M: média; DP: desvio-padrão; IMC: índice de massa corporal; LDL: lipoproteínas de baixa densidade; HDL: lipoproteínas de alta densidade. Fonte: World Health Organization ${ }^{16}$. 
Ao separar os pacientes em grupos observou-se que a maioria já fez (17,5\%) ou está fazendo (42,5\%) acompanhamento dietoterápico com nutricionista do próprio serviço. O uso exclusivo da medicação clozapina (monoterapia) apareceu em $42,5 \%$ dos pacientes, já o restante, $57,5 \%$, faz uso da clozapina juntamente com outros antipsicóticos (politerapia). Grande parte dos pacientes recebe doses altas de clozapina diariamente, sendo que 50,0\% ingerem de $350 \mathrm{mg}$ até $600 \mathrm{mg}$ e $37,5 \%$ ingerem acima de $650 \mathrm{mg}$. Apenas $12,5 \%$ recebem doses de até $300 \mathrm{mg}$.

Na investigação de associações entre parâmetros pode-se observar que a dose de clozapina não apresenta correlação significativa com nenhuma medida antropométrica (Tabela 2). Em relação às variáveis bioquímicas, a dose do fármaco está
Tabela 2. Correlação entre dose de clozapina e parâmetros antropométricos e bioquímicos de pacientes com esquizofrenia. Porto Alegre (RS), 2007.

\begin{tabular}{lcc}
\hline & \multicolumn{2}{c}{ Dose clozapina $(\mathrm{mg})$} \\
\cline { 2 - 3 } & $\mathrm{r}_{\mathrm{s}}$ & $\mathrm{p}$ \\
\hline Dados antropométricos & & \\
IMC $\left(\mathrm{kg} / \mathrm{m}^{2}\right)$ & 0,192 & 0,241 \\
Circunferência abdominal (cm) & 0,211 & 0,197 \\
\% gordura & 0,147 & 0,386 \\
Perfil bioquímico $(\mathrm{mg} / \mathrm{dL})$ & & \\
Colesterol total & & \\
LDL-c & 0,169 & 0,309 \\
HDL-c & 0,391 & 0,025 \\
Triglicerídeos & $-0,046$ & 0,746 \\
Glicemia jejum & 0,084 & 0,612 \\
\hline
\end{tabular}

IMC: índice de massa corporal; LDL: lipoproteínas de baixa densidade; HDL: lipoproteínas de alta densidade. Teste de correlação Spearman.

Fonte: World Health Organization ${ }^{16}$.

Tabela 3. Distribuição percentual de dados antropométricos e alterações bioquímicas em pacientes com diferentes doses de clozapina. Porto Alegre (RS), 2007.

\begin{tabular}{|c|c|c|c|c|}
\hline \multirow{3}{*}{ Variáveis } & \multicolumn{3}{|c|}{ Dose clozapina (mg) } & \multirow{3}{*}{$p$} \\
\hline & \multicolumn{3}{|c|}{$\%$} & \\
\hline & $<300$ & $350-600$ & $>650$ & \\
\hline \multicolumn{5}{|l|}{ Estado nutricional (IMC) } \\
\hline Baixo peso/eutrofia & 50,0 & 30,0 & 20,0 & 0,533 \\
\hline \multicolumn{5}{|c|}{ Circunferência abdominal (cm) } \\
\hline$<94$ & 50,0 & 25,0 & 13,3 & 0,255 \\
\hline$\geq 94$ & 50,0 & 75,0 & 86,7 & \\
\hline \multicolumn{5}{|c|}{ Perfil bioquímico (mg/dL) } \\
\hline \multicolumn{5}{|l|}{$L D L-C$} \\
\hline$<100$ & 75,0 & 57,9 & 14,3 & 0,010 \\
\hline$\geq 100$ & 25,0 & 42,1 & 85,7 & \\
\hline \multicolumn{5}{|l|}{$H D L-C$} \\
\hline$<40$ & 25,0 & 26,3 & 21,4 & 1,000 \\
\hline$\geq 40$ & 75,0 & 73,7 & 78,6 & \\
\hline \multicolumn{5}{|l|}{ Triglicerídeos } \\
\hline$<150$ & 60,0 & 35,0 & 57,1 & 0,519 \\
\hline
\end{tabular}

IMC: índice de massa corporal; Teste exato de Fisher. LDL: lipoproteínas de baixa densidade; HDL: lipoproteínas de alta densidade. Fonte: World Health Organization ${ }^{16}$. 
positivamente correlacionada aos níveis de LDL-C $(r=0,391, p=0,025)$. O tempo de uso de clozapina não se correlaciona com nenhum parâmetro antropométrico ou bioquímico investigado.

Quando os pacientes são divididos em faixas de dose de clozapina (baixas doses: <300mg; doses intermediárias: 350-600mg; e doses altas: $>650 \mathrm{mg}$ ) (Tabela 3), observa-se uma associação significante entre a dosagem e a proporção de LDL-c acima do recomendado $(\geq 100 \mathrm{mg} / \mathrm{dL}$ ). Enquanto $25,0 \%$ pacientes que usam doses inferiores a 300mg apresentam LDL-C $\geq 100 \mathrm{mg} / \mathrm{dL}, 85,7 \%$ dos pacientes que usam doses superiores a $650 \mathrm{mg}$ apresentam $\mathrm{LDL}-\mathrm{c} \geq 100 \mathrm{mg} / \mathrm{dL}$ $(p=0,01)$, o que reforça a associação entre dosagem de clozapina e alterações nos níveis de LDL-c (Tabela 3). Além disso, observou-se que, embora não significante, quanto maior a dose, maior o percentual de pacientes com sobrepeso/obesidade e de circunferência abdominal $\geq 94 \mathrm{~cm}$.

Não houve nenhuma associação significante ao separar os pacientes em relação aos que fazem ou não acompanhamento nutricional.

Independentemente da dosagem, em todas as classificações a maioria dos pacientes apresentou HDL-c elevado, sendo a média de HDL-c encontrada de 44,92mg/dL.

Pacientes que fazem monoterapia não apresentaram diferença em relação a colesterol total, triglicerídeos, LDL-c, HDL-c e circunferência abdominal, ao serem comparados com pacientes em politerapia. Porém, apresentaram percentual maior de níveis de glicose elevada (Figura 1), sendo que os que fazem monoterapia apresentaram $43,80 \%$ de glicemia elevada, enquanto que os que fazem politerapia apresentam $26,10 \%$, embora sem diferença estaticamente significante.

\section{I S C U S S Ã O}

A maioria dos pacientes encontra-se com peso acima dos padrões normais segundo o $\mathrm{IMC}^{16}$,

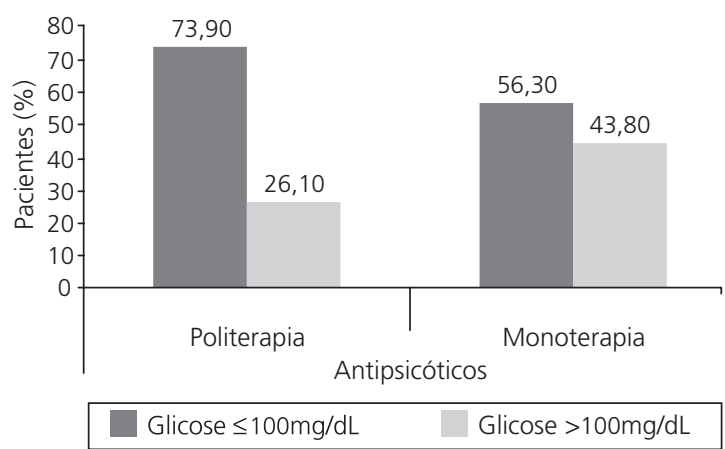

Figura 1. Diferenças na glicemia de jejum em pacientes esquizofrênicos em uso de monoterapia e de politerapia. Porto Alegre (RS), 2007.

Nota: teste Qui-Quadrado; $p=0,420$.

sendo $46,2 \%$ com sobrepeso e $25,6 \%$ com obesidade.

Aproximadamente $23,0 \%$ a $33,5 \%$ da população brasileira apresenta sobrepeso (IMC de 25 a $29,9 \mathrm{~kg} / \mathrm{m}^{2}$ ) e $8,1 \%$ e $12,9 \%$ apresentam obesidade (IMC $\left.>30 \mathrm{~kg} / \mathrm{m}^{2}\right)^{21}$. Observou-se, então, uma freqüência alarmante, tanto de sobrepeso quanto de obesidade, nos pacientes estudados.

A média de IMC destes pacientes foi $27,81 \mathrm{~kg} / \mathrm{m}^{2}$, sugerindo sobrepeso. O mesmo pôde-se observar em estudos semelhantes com esquizofrênicos em uso de antipsicóticos típicos e atípicos, que encontraram média de IMC de $25,27 \mathrm{~kg} / \mathrm{m}^{2}$ e $26,9 \mathrm{~kg} / \mathrm{m}^{2}$ no grupo da clozapina ${ }^{22,23}$. Todos os índices se apresentaram elevados, o que comprova que estes pacientes, geralmente, encontram-se acima do peso recomendado para sua altura. É importante ressaltar que o IMC se correlaciona de forma significativa com alto risco de doenças cardiovasculares ${ }^{24}$.

Estudos distintos, comparando o aumento de peso ocasionado pelo uso de clozapina em relação a outros antipsicóticos, comprovaram que a clozapina foi a responsável pelos maiores índices, sendo o aumento de $7 \%$ a $9 \%$ do peso ou aumento de $4,5 \mathrm{~kg}$ a $6 \mathrm{~kg}^{8,25,26}$. O ganho de peso clinicamente relevante ocorre com freqüência em pacientes em uso de antipsicóticos e um dos principais é a clozapina, que se associa também a uma 
maior incidência de diabetes e dislipidemias, seja devido ao ganho de peso, seja por ação deletéria direta sobre o metabolismo da glicose ${ }^{27}$.

Encontrou-se também circunferência abdominal elevada na maioria dos pacientes, representando um grande risco para o desenvolvimento de doenças cardiovasculares.

Mais da metade dos pacientes pesquisados apresentou percentual de gordura corporal acima de $25 \%$, o que é um risco para doenças associadas à obesidade ${ }^{18}$. Também um grande número apresentou percentual acima da média em um estudo que comprovou a associação da clozapina ao aumento da gordura corporal, em apenas 10 semanas, com pacientes esquizofrênicos ${ }^{28}$.

Os níveis séricos de colesterol total foram avaliados no Brasil em regiões específicas, envolvendo 8045 indivíduos com idade média de 35 anos, no ano de 1998 , onde $38,0 \%$ dos homens e $42,0 \%$ das mulheres estavam com colesterol total acima de $200 \mathrm{mg} / \mathrm{dL}^{17}$. Dados semelhantes foram encontrados nos pacientes portadores de esquizofrenia deste estudo, sendo que $39,5 \%$ apresentaram colesterol total acima de $200 \mathrm{mg} / \mathrm{dL}$.

Neste estudo os pacientes também apresentaram níveis aumentados de triglicerídeos e de LDL-c. A clozapina já tem sido associada à hipertrigliceridemia ${ }^{23}$, porém não a níveis elevados de LDL-C ${ }^{29}$.

Os pacientes que tomam menores doses de clozapina (300mg) são, em geral, mais eutróficos, já os que recebem doses maiores (350mg ou mais) encontram-se com maior proporção de sobrepeso. Estes dados são semelhantes a um estudo segundo o qual, conforme o houve aumento na dose aumentou também o peso, sendo maior em doses acima de $300 \mathrm{mg} / \mathrm{dia}$ e sem nenhuma relação com o sexo dos pacientes ${ }^{30}$.

Observou-se uma associação significante entre a dosagem e a proporção de LDL-c acima do recomendado. Pacientes que usam doses maiores apresentam níveis de LDL-c mais alterados do que os que utilizam doses menores. Este estudo parece confirmar que a clozapina está associada à elevação de LDL-c. Este achado é de grande importância já que em estudos anteriores não foram encontradas alterações nos níveis de LDL-C, independentemente da dose ${ }^{31}$.

O HDL-c esteve elevado em todos os grupos, no presente estudo, independentemente da dose de clozapina. Considerando que os níveis ideais de HDL-c para o sexo masculino são superiores a $40 \mathrm{mg} / \mathrm{dL}$, a clozapina, então, não trouxe alterações nestes parâmetros.

Ao separar os pacientes em grupos distintos quanto aos que fazem uso exclusivo de clozapina, independentemente da dosagem, e os que utilizam outros antipsicóticos concomitantes, não se obtém nenhuma diferença significante. Em relação à glicose, observou-se que os pacientes em monoterapia apresentaram níveis mais elevados, apesar desta diferença não ter significância estatística. Estudos preliminares já evidenciaram o aumento significativo dos níveis de glicose nos pacientes medicados com clozapina ${ }^{23}$.

Pacientes com recente ganho de peso têm baixa auto-estima e uma pobre adaptação psicossocial ${ }^{32}$. É preciso ressaltar que a perda de peso é difícil, mas não é impossível. Uma modificação do comportamento, com auxílio de um programa de dieta e exercícios, é necessária para sustentar a manutenção do peso ${ }^{23}$. Por isso a importância da intervenção nutricional precoce nestes pacientes.

O aumento do peso corporal está relacionado a diversos fatores, tais como: sintomas negativos, pouco acesso a locais para prática de exercícios físicos, situação socioeconômica e exposição aumentada a ambientes de promoção da obesidade. Também influência da genética, mas os mecanismos mais elucidados sugerem que os antipsicóticos podem induzir o aumento de peso em função do aumento do apetite, causando assim um balanço positivo, já que aumenta a energia interna corporal em proporção ao baixo gasto energético ${ }^{33}$.

Recomenda-se que os níveis de colesterol, triglicerídeos e de glicose plasmática de jejum dos 
704 | K. ZORTÉA et al.

pacientes sejam monitorados freqüentemente, sempre avaliando os fatores risco-benefício. Se necessário, um programa de redução de peso deve ser implantado ${ }^{23}$.

\section{CONCLUSÃO}

A maioria dos pacientes encontra-se em sobrepeso, com circunferência abdominal elevada e com percentual de gordura corporal em valores acima dos preconizados, sendo grande parte em quadro de risco para doenças associadas à obesidade. Também apresentam níveis elevados de LDL-c e de triglicerídeos.

O IMC apresentou-se elevado nos pacientes que recebem maiores doses de clozapina. Houve associação significante entre a dosagem de clozapina e o LDL-c. Observou-se que, quanto maior a dose, maior o percentual de pacientes com níveis elevados de sobrepeso/obesidade e de circunferência abdominal, embora não significante. O colesterol total e a glicose não tiveram associação positiva em relação à dose de clozapina.

Não houve associação entre acompanhamento nutricional nem monoterapia de clozapina, em relação aos parâmetros avaliados no estudo.

Os resultados suportam hipóteses de que pacientes tratados com clozapina apresentam significante aumento de peso e anormalidades lipídicas, necessitando de monitoramento freqüente dos níveis plasmáticos e intervenção nutricional precoce, visando à otimização do tratamento.

\section{COLABORADORES}

K. ZORTÉA e P.M. BOCK participaram da concepção e do desenho do estudo, da análise, da interpretação dos dados e da redação do manuscrito. P.S.B. ABREU participou da análise e da interpretação dos dados. D.B. MORENO participou da concepção e do desenho.

\section{REFER Ê NCIAS}

1. Manual Diagnóstico e Estatístico de Transtornos Mentais - DSM-IV-TR. 4a. ed. Porto Alegre: Artmed; 2003.

2. Organização Mundial da Saúde. Classificação de transtornos mentais e de comportamento da CID-10: descrições clínicas e diretrizes diagnósticas. Porto Alegre: Artmed; 1993.

3. Mari JJ, Leitão RJ. A epidemiologia da esquizofrenia. Rev Bras Psiquiatr. 2000; 22(1):15-7. doi: 10.1590/S1516-44462000000500006.

4. Rang HP, Dale MM, Ritter JM, Moore PK. Farmacologia. 5a. ed. Rio de Janeiro: Elsevier; 2004.

5. Kaplan HI. Compêndio de psiquiatria: ciências do comportamento e psiquiatria clínica. 7a. ed. Porto Alegre: Artmed; 1997.

6. Chaves AC. Diferenças entre os sexos na esquizofrenia. Rev Bras Psiquiatr. 2000; 22(1):21-2. doi: 10.1590/S1516-44462000000500008.

7. Gama CS, Souza CM, Lobato MI, Abreu PSB. Clozapine use report in 56 patients seen by Clerkship of Health and Environment of the State of Rio Grande do Sul's Program of Attention to the Refractory Schizophrenia. Rev Psiquiatr Rio Grande Sul. 2004; 26(1):21-8. doi: 10.1590/ S0101-81082004000100004.

8. Morais RMO, Oliveira IR. Antipsicóticos. In: Silva P. Farmacologia. 7a. ed. Rio de Janeiro: Guanabara Koogan; 2006.

9. Casey DE. Dyslipidemia and atypical antipsychotis drugs. J Clin Psychiatry. 2004; 65(18):27-35.

10. Lewis SW, Barnes TRE, Davies L, Murray RM, Dunn G, Hayhurst KP, et al. Randomized controlled trial of effect of prescription of clozapine versus other second-generation antipsychotic drugs in resistant schizophrenia. Schizophr Bull. 2006; 32(4): 715-23.

11. Meltzer, HY. Putting metabolic side effects into perspective: risks versus benefits of atypical antipsychotics. J Clin Psychiatry. 2001; 62(27):35-9.

12. Munoz P, Gallardo R. Transtornos metabólicos en pacientes esquizofrênicos tratados con clozapina. Rev Chil de Neuro-Psiquiatr. 2004; 42(1):37-42. doi: $10.4067 /$ S0717-92272004000100005.

13. Brasil. I Diretriz Brasileira de Diagnóstico e Tratamento da Síndrome Metabólica. Arq Bras Cardiol. 2005; 84(1):3-28. doi: 10.1590/S0066-782X2005 000700001 .

14. Duncan BB, Schmidt MI. Chronic activation of the innate immune system may underlie the metabolic syndrome. São Paulo J Med. 2001; 119(3):122-7. doi: 10.1590/S1516-31802001000300008. 
15. Goff DC, Cather C, Evins AE, Henderson DC, Freudenreich O, Copeland PM, et al. Medical morbidity and mortality in Schizophrenia: guidelines for psychiatrists. J Clin Psychiatry. 2005; 66(2):183-93.

16. World Health Organization. Physical status: the use and interpretation of anthropometry. Genebra: WHO; 1998.

17. Brasil. IV Diretriz Brasileira Sobre as Dislipidemias e Prevenção da Aterosclerose do Departamento de Aterosclerose da Sociedade Brasileira de Cardiologia. Arq Bras Cardiol. 2007; 88(1)2-19. doi: 10.1590/S0066-782X2007000700002.

18. Lohman TG. Advances in body composition assessment. Current issues in exercise science series. Monografh n.3. Champaign (IL): Human Kinetics; 1992.

19. Executive Summary of the Third Report of the National Cholesterol Education Program (NCEP). Expert Panel on Detection, Evaluation, and Treatment of High Blood Cholesterol in Adults (Adult Treatment Panel III). JAMA 2001; 285(19): 2486-97.

20. American Diabetes Association. Standards of medical care in diabetes. Diabetes Care. 2005; 28(1):4-36.

21. Brasil. Ministério da Saúde. Guia alimentar para a população brasileira: promovendo a alimentação saudável. Brasília; 2006.

22. Souza VBN, Guedes ARA. Índice de massa corpórea de esquizofrênicos em uso de antipsicóticos típicos e atípicos. Neurobiologia. 2002; 65(1):77-80.

23. Henderson DC, Cagliero E, Gray C, Nasrallah RA, Hayden DL, Schoenfeld DA, et al. Clozapine, diabetes mellitus, weight gain, and lipid abnormalities: a five-year naturalistic study. Am J Psychiatry. 2000; 157(6):975-81.

24. Cercato C, Mancini MC, Arguello AMC, Passos VQ, Villares SMF, Halpern A. Systemic hypertension, diabetes mellitus, and dyslipidemia in relation to body mass index: evaluation of a Brazilian population. Rev Hosp Clin. 2004; 59(3):113-8. doi: 10.1590/S0041-87812004000300004.
25. Bustillo JR, Buchanan Robert W, Irish D, Breier A. Differential effect of Clozapine on weight: a controlled study. Am J Psychiatry.1996; 153(6): 817-9.

26. Allison DB, Mentore JL, Ed MS, Heo M, Chandler LP, Cappelleri JC, et al. Antipsychotics-induced weight-gain: a comprehensive research synthesis. Am J Psychiatry. 1999; 156(11):1686-9.

27. Teixeira PJR, Rocha FL. Efeitos adversos metabólicos de antipsicóticos e estabilizadores de humor. Rev Psiquiatr Rio Grande do Sul. 2006; 28(2):186-96. doi: 10.1590/S0101-81082006000200011.

28. Kivircik BB, Alptekin K, Çaliskan S, Çomlejçi A, Oruk $\mathrm{G}$, Tumuklu $\mathrm{M}$, et al. Effect of clozapine on serum leptin, insulin levels, and body weight and composition in patients with schizophrenia. Prog Neuro-Psychopharmacol Biol Psychiatry. 2003; 27(5):795-9.

29. Wu MK, Huang CY, Liou YJ, Wang CK, Lee SD. Glucose-insulin homeostasis, lipid profiles and GH-IGF-IGFBP axis in clozapine-treated schizophrenic obesity versus non-psychiatric obesity. Int J Obes. 2008; 32(3):436-42. doi:10.1038/sj.ijo.0803750.

30. Leon, J, Diaz FJ, Josiassen RC, Cooper TB, Simpson GM. Weight gain during a double-blind multidosage Clozapine study. J Clin Psychopharmacol. 2007; 27(1):22-7.

31. Baymiller SP, Ball P, McMahon RP, Buchanan RW. Serum glucose and lipid changes during the course of clozapine treatment: the effect of concurrent beta-adrenergic antagonist treatment. Schizophr Res. 2003; 59(1):49-57.

32. De Hert, M, Peuskens B, Winkel RV, Kalnicka D, Hanssens L, Eyck DV, et al. Body weight and selfesteem in patients with schizophrenia evaluated with B-WISE ${ }^{\circledR}$. Schizophr Res. 2006; 88(1-3): 222-6.

33. McIntyre RS. Mechanisms of antipsychotic-induced weight gain. J Clin Psychiatry. 2001; 62(23):23-9.

Recebido em:14/11/2007

Versão final reapresentada em: 13/8/2008

Aprovado em: 9/6/2009 